\title{
Osteogenic differentiation of two distinct subpopulations of human adipose-derived stem cells: an in vitro and in vivo study
}

\author{
T. Rada ${ }^{1,2}$, T. C. Santos ${ }^{1,2,3}$, A. P. Marques ${ }^{1,2}$, V. M. Correlo ${ }^{1,2}$, A. M. Frias ${ }^{1,2}$, A. G. Castro ${ }^{3}$, \\ N. M. Neves ${ }^{1,2}$, M. E. Gomes ${ }^{1,2 *}$ and R. L. Reis ${ }^{1,2}$ \\ ${ }^{1} 3$ Bs Research Group - Biomaterials, Biodegradables and Biomimetics, Department of Polymer Engineering, University of Minho, \\ Headquarters of the European Institute of Excellence on Tissue Engineering and Regenerative Medicine, AvePark, Guimarães, Portugal \\ ${ }^{2}$ Institute for Biotechnology and Bioengineering (IBB), PT Government Associated Laboratory, Portugal \\ ${ }^{3}$ Institute of Health and Life Sciences, School of Health Sciences, University of Minho, Campus de Gualtar, 4710-057 Braga, Portugal
}

\begin{abstract}
The first stem cells considered for the reconstruction of bone were bone marrow mesenchymal stem cells (BMSCs). Subsequently, cells with similar marker expression panel and differentiation potential were found in new sources of cells, such as adipose tissue. This source of stem cells has a promising future in tissue-engineering applications, considering the abundance of this tissue in the human body, the easy harvesting and the high number of stem cells that are available from such a small amount of tissue. The isolation of the adipose stem cells is generally performed by means of enzymatic digestion of the tissues, followed by a natural selection of the stem cells based on their capacity to adhere to the culture flasks, leading to a quite heterogeneous population. This constitutes a major drawback for the use of these cells, since the heterogeneity of the cell culture obtained can compromise their proliferation and differentiation potential. In the present study we have analysed the in vitro and in vivo behaviour of two selected subpopulations with high osteogenic potential. For this purpose, ASCs ${ }^{\mathrm{CD} 29+}$ and $\mathrm{ASCs}^{\text {STRO-1+ }}$ subpopulations were isolated and in vitro cultured onto a biodegradable polymeric scaffold, using osteogenic medium, before implantation in a nude mice model. The biodegradable polymeric scaffold used is a fibre-mesh structure based on a blend of starch and polycaprolatone (SPCL) that has been successfully used in several bone tissue-engineering studies. The implanted ASCs-scaffold constructs promoted the formation of new bone tissue in nude mice. However, the results obtained show differences in the behaviour of the two ASCs subpopulations under study, particularly regarding their potential to differentiate into the osteogenic lineage, and allowed the indentification of ASCs ${ }^{\text {STRO-1+ }}$ as the best subpopulation for bone tissue-engineering applications. Copyright $\odot 2011$ John Wiley \& Sons, Ltd.
\end{abstract}

Received 14 October 2010; Accepted 28 October 2010

Keywords adipose stem cells; osteogenic differenctiation; bone; stem cells; SPCL; in vivo

\section{Introduction}

*Correspondence to: M. E. Gomes, 3Bs Research GroupBiomaterials, Biodegradables and Biomimetics, Department of Polymer Engineering, University of Minho, Headquarters of the European Institute of Excellence on Tissue Engineering and Regenerative Medicine, AvePark, Guimarães, Portugal.

E-mail: megomes@dep.uminho.pt
Bone tissue engineering is that discipline of regenerative medicine that involves the development of therapies for the regeneration of skeletal tissue using cells and a support material that promotes the adhesion, growth and differentiation of cells, sometimes also with the help of specific growth factors. 
The scaffold is a fundamental element of bone tissueengineering strategies, since it is very important to provide bone precursor cells with an appropriate threedimensional (3D) support and adequate biomechanical environment. Therefore, the materials selected for the scaffold should allow higher control to be achieved over parameters such as porosity, pore size, surface area and the mechanical properties of the 3D support (Silva et al., 2007), and simultaneously they should be degradable and biocompatible (Gomes and Reis, 2004). Several studies (Gomes et al., 2001a, 2001b, 2003, 2006a, 2006b; Tuzakoglu et al., 2005, 2009) have demonstrated that fibre-meshes obtained from a blend of starch and polycaprolactone (SPCL) are an excellent scaffolding material, that allows the proliferation and osteogenic differentiation of stem cells and also the adhesion and growth of endothelial cells, providing evidence of their suitability to support the formation of vascularized bone-like tissue (Fuchs et al., 2009a, 2009b; Santos et al., 2007, 2008). SPCL-based materials were therefore the scaffolds selected for the present study.

In bone tissue-engineering applications, the adult stem cells that have been most widely used are mesenchymal stem cells (MSCs). The first and most well-known source of MSCs is the bone marrow. Lately, several research studies (Yoshimura et al., 2007; Gay et al., 2007; Hennrick et al., 2007; Hui et al., 2005; Majore et al., 2009) have shown that MSCs can be isolated from other sources, such as adipose tissue. Although the discovery of the adipose tissue as source of stem cells is relatively recent (Zuk et al., 2001), the high potential of these stem cells for tissue-engineering applications and other cell-based therapies is very well supported by the simplicity of the procedures to harvest adipose tissue, which causes minimal discomfort for patients while allowing large amounts of tissue to be obtained, from which it is possible to isolate a large quantity of stem cells. Furthermore, adipose stem cells (ASCs) can be differentiated into a large number of cell types, such as chondrocytes and osteoblasts (Xu et al., 2005; Ogawa et al., 2004; Zuk et al., 2002; Huang et al., 2005; Mochizuki et al., 2006), neuronlike cells (Zuk et al., 2001; Safford et al., 2002; Fujimura et al., 2005), myocytes (Lee and Kemp, 2006; Fraser et al., 2006; Strem et al., 2005; Planat-Bernard et al., 2004), hepatocytes (Yamamoto et al., 2008; Stock et al., 2008) and, of course, adipocytes (Rada et al., 2009a; Gimble and Guilak, 2003a, 2003b). Additionally, the ASCs have a low immunological reactivity, as demonstrated by recent studies where, in fact, the ASCs were found to be negative regarding the expression of the most important immunologically relevant surface antigens, such as MHCII, CD40, CD40L, CD80 and CD86. These cells also do not express IL-10 and TGF $\beta$, well-known inhibitor factors of lymphocytes (Puissant et al., 2005) and this lack of expression is also maintained after osteogenic differentiation (McIntosh et al., 2006). ASCs are also unable to incite a response of allogenic lymphocytes in vitro, inhibiting allogenic lymphocyte proliferation induced by allogenic PBMCs or mitogens (McIntosh et al., 2006), and this phenomenon is proportional to the number of ASCs and to the time of contact between stem cells and lymphocytes (Rada et al., 2009a; Puissant et al., 2005).

In previous studies (Rada et al., 2009b, 2010) we have isolated ASC subpopulations (from rat and human origin) using immunomagnetic beads coated with a range of antibody (ab) markers for these stem cells, viz. CD29, CD105, CD90, p75, STRO-1, CD44, CD73 and CD49d. From the results obtained in these studies, it was possible to select two subpopulations with high osteogenic potential, viz. the subpopulations isolated using immunomagnetic beads coated with anti STRO-1 $\mathrm{ab}$ and CD29 ab.

Although in previous studies our group was able to select ASC subpopulations with good osteogenic differentiation potential, all the experiments were carried out in vitro; obviously between an in vitro and an in vivo system there are several differences that could affect the behaviour and the differentiation potential of the cells. Since the final aim of the studies including cells and scaffolds is an application in vivo, the main aim of this study was to assess the potential of these two cell subpopulations in vivo and eventually select the most adequate hASCs subpopulation to use in bone tissue-engineering strategies. For this purpose, the selected hASCs subpopulations were seeded onto starch-polycaprolactone (SPCL) fibre-mesh scaffold, a scaffold that has been well studied by our research group (Gomes et al., 2003, 2006b, 2008; Tuzakoglu et al., 2009; Fuchs et al., 2009a; Santos et al., 2007; Oliviera et al., 2007; da Silva et al., 2009; Martins et al., 2008, 2009; Silva et al., 2009). The cells used were transfected with green fluorescent protein (GFP) to assess their presence in the scaffold for the whole time of the experiments. The cell-scaffold constructs were cultured in vitro for 1 day with osteogenic medium and then implanted in an ectopic site (subcutaneous) in nude mice. The constructs were retrieved 6 weeks after implantation and analysed by RT-PCR and histologically, as well as by micro-computerized tomography ( $\mu \mathrm{CT}$ ), to assess hASCs differentiation and subsequent new bone formation.

\section{Materials and methods}

\subsection{Scaffold preparation}

Scaffolds based on SPCL (a 30:70 wt\% blend of starch with poly-caprolactone) were prepared by a fibre-bonding process consisting of cutting and sintering melt-spun fibres with a diameter of approximately $180 \mu \mathrm{m}$, as described previously (Gomes et al., 2006b; Santos et al., 2008; Martins et al., 2008). All samples were then cut into discs of approximately $6 \mathrm{~mm}$ diameter and $1.5-2 \mathrm{~mm}$ height and sterilized using ethylene oxide. In the SPCL fibre-meshes obtained by this method the porosity of the 
scaffolds was 75\% and highly interconnected (Gomes et al., 2003).

\subsection{Adipose tissue digestion and cell harvesting}

Human subcutaneous adipose tissue samples were obtained from lipoaspiration procedures performed in women within an age range of 35-45 years, under a previously approved protocol with a local hospital (Hospital da Prelada, Porto, Portugal).

The tissue was first washed with phosphate-buffered saline (PBS; Sigma-Aldrich, Germany) containing $5 \mathrm{mg} /$ $500 \mathrm{ml}$ Ciprobay 200 (Bayer) and 10\% AntibioticAntimycotic (Gibco, UK) and then digested with a $0.2 \%$ collagenase type I A (Sigma-Aldrich) solution in PBS for $60 \mathrm{~min}$ at $37^{\circ} \mathrm{C}$ under gentle stirring. The digested tissue was filtered using a $100 \mu \mathrm{m}$ filter mesh (Sigma-Aldrich), centrifuged at $1250 \mathrm{rpm}$ for $7 \mathrm{~min}$ at $20^{\circ} \mathrm{C}$ and then the supernatant was eliminated. The cell pellet obtained was resuspended and further submitted to a procedure for isolating cell subpopulations, as described in the next section.

\subsection{Isolation of hASC subpopulations}

For this study we selected two subpopulations isolated by a method based on the use of immunomagnetic beads, which has been described previously (Rada et al., 2009b). The method consists of using immunomagnetic Dynal M450 Epoxy beads (Dynal Biotech, USA) that were coated with either anti CD29 or STRO-1 antibody by preparing a mixture at a concentration of $4 \mu \mathrm{g}$ ab/ $1 \times 10^{7}$ Dynabeads. The CD 29 and STRO-1 were selected within a range of antibodies based on a previous study (Rada et al., 2010), which showed that the cells isolated with these two antibodies exhibit a high osteogenic differentiation potential. The cell pellet obtained from the enzymatic digestion of adipose tissue was resuspended in $0.1 \%$ BSA (Sigma-Aldrich) in PBS and then mixed with the Ab-coated Dynabeads. The mixture was incubated for $30 \mathrm{~min}$ at $4{ }^{\circ} \mathrm{C}$ under gentle stirring and subsequently the cell suspension and beads mixture was washed with PBS and BSA. Finally, the cells bonded to the Abcoated Dynabeads were separated from the remainder of the cell suspension using the Dynal MPC magnet (Dynal Biotech) and seeded in $25 \mathrm{~cm}^{2}$ cell culture flasks.

\subsection{Cell transfection of hASCs}

The isolated hASCs subpopulations were transfected with green fluorescent protein (GFP) in order to enable these cells to be traced upon implantation and their role in the formation of new tissue in vivo to be analysed. For this purpose, after reaching confluence, the cell culture medium was substituted by medium without antibiotic and without serum. On the following day, lipofectamine (Invitrogen, USA) and pDsGreen-N1 Vector (Clontech, USA) were used to transfect the cells. Briefly, $8 \mu \mathrm{g}$ plasmid DNA was diluted in $0.5 \mathrm{ml}$ DMEM medium without FBS and $20 \mu \mathrm{l}$ lipofectamine 2000 were diluted in $0.5 \mathrm{ml}$ DMEM serum-free medium. The two solutions were then mixed together and added to cells in the $25 \mathrm{~cm}^{2}$ culture flasks containing $5 \mathrm{ml}$ of DMEM without antibiotic. The cells were exposed to the complex of lipofectamine and plasmid (in an incubator at $37^{\circ} \mathrm{C}$ and $5 \% \mathrm{CO}_{2}$ ) for $4 \mathrm{~h}$ and then the medium was substituted with DMEM containing 1\% antibiotic (Sigma-Aldrich) and $10 \%$ FBS.

\subsection{Cell seeding and culturing into SPCL scaffolds}

After transfection and further expansion for about 15 days, the $\mathrm{CD}^{2} 9^{+}$and STRO-1 ${ }^{+}$ASCs populations were trypsinized, counted and seeded/cultured in SPCL fibre-mesh scaffolds. The scaffolds were press-fitted into cassettes for a higher cell-seeding efficiency, and placed in six-well plates; then $300 \mu \mathrm{l}$ of a diluted cell suspension containing $5 \times 10^{6}$ cells was seeded onto the surface of each scaffold. The well plate was then transferred to the incubator for $3 \mathrm{~h}$ and afterwards $1.5 \mathrm{ml}$ DMEM was added to each well containing the cassette with the cell-seeded scaffold. After $12 \mathrm{~h}$ of incubation, the scaffolds were gently removed from the cassettes and transferred into new six-well plates, adding $1 \mathrm{ml}$ basal medium. Each ASC subpopulation was seeded in 14 scaffolds: eight to be implanted in nude mice and six that were used as in vitro controls. The two subpopulations of ASCs were also seeded in six-well plates and kept in culture for the same period of the in vitro/in vivo experiment.

After 1 day of culture, the culture medium was replaced by osteogenic medium composed of $\alpha$-modified Eagle's medium (Sigma-Aldrich) supplemented with $50 \mu \mathrm{g} / \mathrm{ml}$ ascorbic acid (Sigma-Aldrich), $10^{-8} \mathrm{M}$ dexamethasone (Sigma-Aldrich), $10 \mathrm{~mm} \beta$-glycerol phosphate (SigmaAldrich), 10\% FBS (Gibco) and 1\% Antibiotic/Antimycotic (Gibco). The cell-seeded scaffolds were cultured with osteogenic medium for 2 weeks, except one group of the in vitro control which was cultured with basal medium through the whole experiment.

\subsection{In vivo implantation of the cell scaffold construct}

For the in vivo experiments, six female nude mice aged 4 weeks (Harlan Laboratories, USA) were used. Each test animal was anaesthetized with a subcutaneous injection of $0.5 \mathrm{mg} / \mathrm{kg}$ medetomidine hydrochloride and $75 \mathrm{mg} / \mathrm{kg}$ ketamine hydrochloride. Under sterile conditions (vertical laminar flow chamber), the skin was disinfected and two medial and ventral incisions (approximately 
$1 \mathrm{~cm}$ ) were performed in the dorsum of each mouse, containing the subcutis and the panniculus carnosus (skin smooth muscle). Craniolaterally orientated pockets (two per incision) were subcutaneously created by blunt dissection. The scaffolds (four samples/animal) were inserted into these pockets The panniculus carnosus and the skin were carefully sutured and the wounds desinfected. The animals were kept with food and water ad libitum during the whole time of implantation.

At the end point of the experiment ( 6 weeks), the animals were sacrificed by cervical dislocation. The implants and respective surrounding tissue were explanted and processed for histological evaluation and molecular biology analysis by real-time reverse transcriptase polymerase chain reaction (RT-PCR) and $\mu \mathrm{CT}$, as described in further sections.

\subsection{RT-PCR}

The mRNA of each sample was extracted with TriZol reagent (Invitrogen), following the procedure provided by the supplier. Briefly, $800 \mu 1$ TriZol was added for each sample. After an incubation of $5 \mathrm{~min}$, an additional $160 \mu \mathrm{l}$ of chloroform (Sigma-Aldrich) was added; the samples were then incubated for $15 \mathrm{~min}$ at $4{ }^{\circ} \mathrm{C}$ and centrifuged at the same temperature and $13000 \mathrm{rpm}$ for $15 \mathrm{~min}$. After the centrifugation, the aqueous part was collected and an equal part of isopropanol (Sigma-Aldrich) was added. After an incubation of $2 \mathrm{~h}$ at $-20^{\circ} \mathrm{C}$ the samples were washed in ethanol, centrifuged at $4{ }^{\circ} \mathrm{C}$ and $9000 \mathrm{rpm}$ for $5 \mathrm{~min}$ and resuspended in $12 \mu \mathrm{l}$ RNase/DNase-free water (Gibco). The samples were quantified using a ND1000 Spectrophotometer (NanoDrop Technologies, USA). The samples selected for the cDNa synthesis were those presenting a 260:280 ratio in the range 1.7-2.0. The cDNA synthesis was performed using the iScript cDNA synthesis Kit (BioRad, USA), and the MiniOpticom realtime PCR Detection System (BioRad), using an initial amount of $2 \mu \mathrm{g}$ mRNA and a total volume of $20 \mu \mathrm{l}$. RNAse-free water (Gibco) was used as the negative control.

\subsection{Real-time $P C R$}

The expression of RunX-2, Osterix, Osteopontin and Osteocalcin was assessed on each sample after the mRNA extraction and the RT-PCR synthesis as described above for the synthesis of the cDNA.

For each sample, GAPDH was used as housekeeping gene. The primers were previously designed using Primer 3 software (v. 0.4.0) and synthesized by MWG Biotech (Germany).

The real-time PCR was carried out using iQ Syber Green SuperMix equipment (BioRad, USA). The procedure followed to perform the reactions was based on the manufacturer's instructions. The data obtained was normalized using the ${ }^{\Delta \Delta} \mathrm{Ct}$ method. The results obtained were elaborated using Origin Pro 7.0 software (OriginLab Corp., USA) and the correlation between the dataset from the same gene analysed was investigated using the single-factor ANOVA method.

\subsection{Histological analysis}

The samples harvested from the in vivo and in vitro experiments were fixed in formalin, paraffin embedded and then sectioned at $4 \mu \mathrm{m}$ thickness using a Microm HM 355S microtome (Thermo Scientific, USA). Sample sections were floated in a water bath at $40^{\circ} \mathrm{C}$, placed on microscope slides, dewaxed in xylenes and rehydrated in an ethanol bath and then stained or immunostained as follows:

- Alizarin red staining. Sections were stained with a $2 \%$ alizarin red solution (Merck, Germany) in distilled water for $5 \mathrm{~min}$, and finally washed with distilled water.

- Xylenol orange staining. Sections were stained for 5 min with a solution previously prepared by dissolving powder stain in distilled water and the $\mathrm{pH}$ was adjusted to 7.2-7.4 using $\mathrm{HCl}$.

- Immunohistological analysis. For the immunohistological analysis (collagen I and osteopontin) the Vectastain ABC Kit (Vector Laboratories, USA) was used. The sample sections were washed with distilled water and then incubated for $30 \mathrm{~min}$ in $0.3 \% \mathrm{H}_{2} \mathrm{O}_{2}$-methanol solution to quench endogenous peroxidase activity. After this step, the slides were washed with PBS for $5 \mathrm{~min}$ and afterwards incubated for 30 min with diluted normal serum. Then the sections were washed again and incubated for $30 \mathrm{~min}$ at room temperature with the primary antibody under analysis, viz. anti-collagen I antibody (Santa Cruz Biotechnologies, USA) and anti-osteopontin antibody (AbCam, USA). After this incubation period the sections were incubated for $30 \mathrm{~min}$ at room temperature with diluted biotinylated secondary antibody solution. Finally the sections were coloured using the DAB substrate Kit (Vector Laboratories, USA). To observe the localization of hASC implanted cells (transfected with GFP), further sections were observed under a fluorescence microscope within $12 \mathrm{~h}$ after the collection of the implants from the mice.

- Microscopical analysis. All stained/immunostained samples were observed under an Imager. Z1 fluorescence microscope (Zeiss, Germany) and photographed using an Axio Cam MRm (Zeiss).

\subsection{Micro-computerized tomography $(\mu \mathrm{CT})$ analysis}

The formation of mineralized tissue in the explants was evaluated using a high-resolution $\mu$ CT Skyscan 1072 

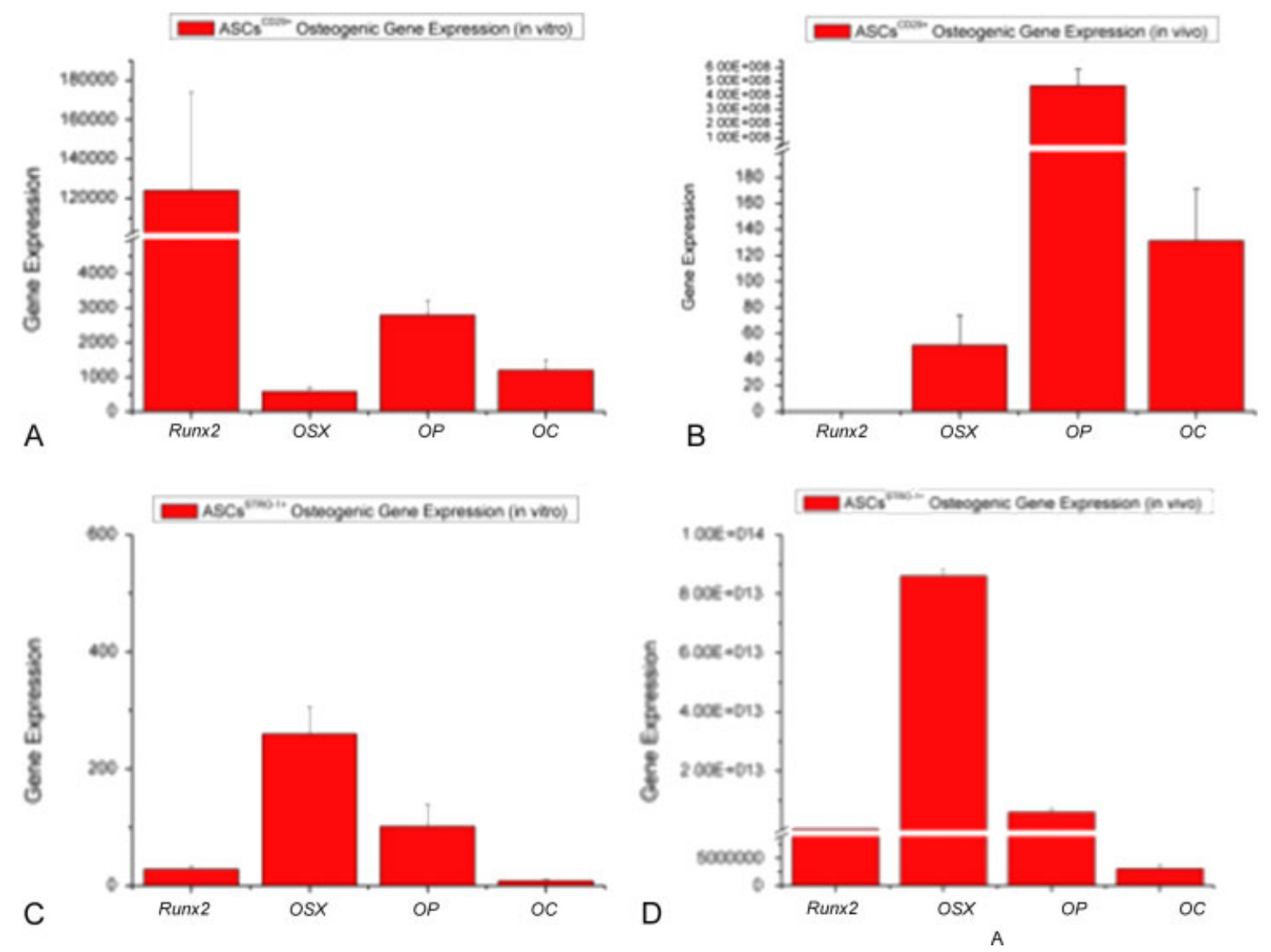

Figure 1. Graphic representation of the results obtained from real-time RT-PCR analysis of runx-2, osterix (OSX), osteopontin (OP) and osteocalcin $(O C)$ obtained in the constructs seeded/cultured with the two hASCs populations considered and either implanted or in vitro cultured under different conditions. (A) ASCs ${ }^{\mathrm{CD} 29+}$ seeded in SPCL scaffolds and cultured in vitro with osteogenic medium; (B) ASCs ${ }^{\mathrm{CD} 29+}$ seeded in SPCL scaffolds and implanted in nude mice; (C) ASCs ${ }^{\text {SRO-1+ }}{ }^{-}$seeded in SPCL scaffolds and cultured in vitro with osteogenic medium; (D) ASCs ${ }^{\text {SRO }-1+}$ seeded in SPCL scaffolds and implanted in nude mice

scanner (Skyscan, Kontich, Belgium). Each sample was scanned in high-resolution mode with a pixel size of $8.7 \mu \mathrm{m}$ and an exposure time of $1.8 \mathrm{~ms}$. The X-ray source was set at $51 \mathrm{keV}$ of energy and $169 \mu \mathrm{A}$ of current. Approximately 400 projections were acquired over a rotation of $180^{\circ}$ with a rotation step of $0.45^{\circ}$. Each one of these projections was segmented into binary images with a dynamic threshold of 220-255 (grey values) to assess new bone formation. These datasets were used for morphometric analysis (CT Analyser, v. 1.5.1.5, SkyScan) and to built 3D models (ANT 3D creator, v2.4, SkyScan). The distribution and quantification of new bone formation in the scaffolds was assessed by a $3 \mathrm{D}$ virtual model that was created, visualized and registered using both image processing softwares (CT Analyser and ANT 3D creator).

\subsection{Statistical analysis}

Data obtained from real-time RT-PCR analysis are presented as arithmetic means \pm SD. An ANOVA singlefactor test was used to compare the mean values and differences were considered to be statistically significant at $p<0.05$. All statistical analyses were performed using Origin Pro 7.0 software (OriginLab Corp., USA).

\section{Results}

\subsection{ASCs gene expression}

Real-time RT-PCR was used to analyse the expression of runx-2, osterix, osteopontin and osteocalcin in the samples retrieved from the in vivo implantation and from the in vitro cultures carried out.

The results obtained from real-time RT-PCR analyses are represented in the graphics presented in Figure 1. In general, these results showed that in all the conditions studied, the expression of the osteogenic markers considered is observed. However, in the cell-scaffolds cultured in vitro, the gene expression levels differ significantly with respect to the cells that were implanted in the nude mice. In fact, concerning $\mathrm{ASC}^{\mathrm{CD} 29+}$, the expression of osteopontin is much higher in implanted cells than in the cells cultured in vitro, while concerning runx-2 expression the opposite behaviour was observed. For the ASC ${ }^{\mathrm{CD} 29+}$ subpopulation, the expression of the other genes considered is not considerably different in the in vivo as compared to the in vitro samples. On the other hand, in samples corresponding to the $\mathrm{ASC}^{\mathrm{STRO}-1+}$ subpopulation, all the genes considered are overexpressed in the samples retrieved from nude mice compared with those 


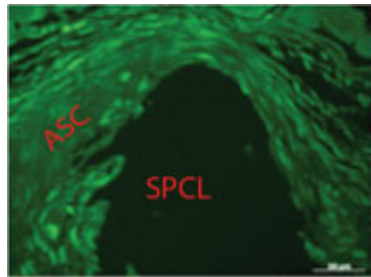

A

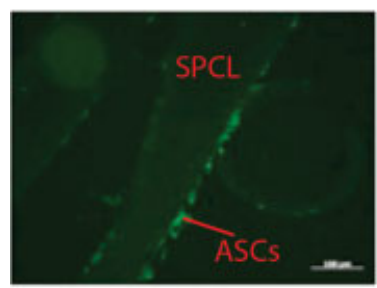

D

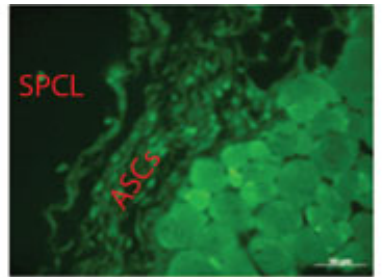

B

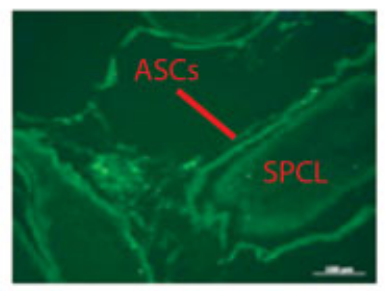

$\mathrm{E}$

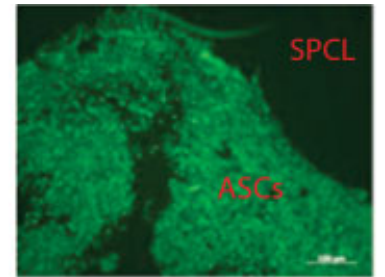

C

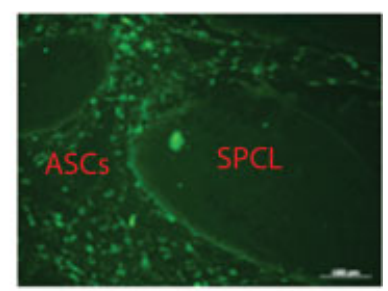

F

Figure 2. Fluorescent microscope images of the constructs seeded/cultures with the two hASCs populations considered transfected with GFP and either implanted or in vitro cultured under different conditions. (A) ASCs ${ }^{\mathrm{CD} 29+}$ seeded in SPCL scaffolds and implanted in nude mice; (B) ASCs ${ }^{\text {STRO-1+ }}$ seeded in SPCL scaffolds and implanted in nude mice; (C) ASCs ${ }^{\mathrm{CD} 29+}$ seeded in SPCL scaffolds and cultured in vitro with basal medium; (D) ASCs ${ }^{\text {STRO-1+ }}$ seeded in SPCL scaffolds and cultured in vitro with basal medium; (E) ASCs ${ }^{\mathrm{CD} 29+}$ seeded in SPCL scaffolds and cultured in vitro with osteogenic medium; (F) ASCs ${ }^{\text {TRO }-1+}$ seeded in SPCL scaffolds and cultured in vitro with osteogenic medium

cultured in vitro. Most importantly, real-time RT-PCR results clearly showed that the ASC $\mathrm{STRO}^{-1+}$ population exhibited a gene expression of all the osteogenic markers analysed higher than the ASC ${ }^{\mathrm{CD} 29+}$ population.

\subsection{Localization of hASCs - green fluorescent protein detection}

The samples retrieved from the in vivo experiment and those cultured in vitro during the same time period (with either basal medium or with osteogenic medium) were observed under a fluorescence microscope, aiming to detect the ASCs producing GFP and therefore to relate the presence (or not) of cells that were originally seeded/implanted to the functionality of the cell-scaffold constructs. In all the ASCs-scaffold samples considered, containing either $\mathrm{ASC}^{\mathrm{CD} 29}$, or $\mathrm{ASC}^{\mathrm{STRO}-1+}$ and implanted or cultured in vitro, it was possible to detect cells that were initially seeded into the scaffolds (Figure 2). The presence of the transfected cells within the scaffolds that are implanted in vivo demonstrate that most of the cells did not migrate during the 6 weeks of the in vivo implantation and that all the results obtained are related to the human ASCs that were originally implanted.

\subsection{Alizarin red and xylenol orange staining}

After the 6 weeks of implantation/in vitro culture, all the samples were positively stained with alizarin red, as shown in Figure 3, indicating the occurrence of a mineralization process. In all the study groups it was also possible to visualize positive staining with xylenol orange, showing the occurrence of mineralization in all cell-scaffold constructs considered (Figure 4). However, the samples that were implanted exhibited a higher fluorescence, indicating a more extensive mineralization than in constructs that were cultured in vitro for the same period of time.

\subsection{Immunohistological analysis}

All samples retrieved after 6 weeks of implantation/in vitro culture were immunostained for collagen I and osteopontin (Figures 5, 6). The results obtained are in good agreement with those obtained from real-time RT-PCR analysis: in the cell-scaffold constructs cultured in vitro with basal medium, there are no signs of the presence of the markers assessed, suggesting that, under these conditions, the ASCs did not differentiate into osteoblastlike cells; in the cell-scaffold constructs in vitro cultured with osteogenic medium, a light coloration was observed, indicating that the process of osteogenic differentiation might be ongoing; finally, the hASC-scaffold constructs retrieved from the in vivo implantation showed strong positive staining regarding both osteogenic markers considered in the immunohistological assay.

\subsection{Micro-computerized tomography ( $\mu$-CT) analysis}

In Figure 7 it is possible to visualize two representative image of $\mathrm{ASC}^{\mathrm{STRO}-1+}$ - scaffold and $\mathrm{ASC}^{\mathrm{CD} 29+}$ - scaffold constructs retrieved from the in vivo experiments and analysed by $\mu \mathrm{CT}$, that show very clearly that in constructs with $\mathrm{ASCs}^{\mathrm{STRO}-1+}$ the mineralization is much more evident than in constructs seeded with $\mathrm{ASCs}^{\mathrm{CD} 29+}$. The presence of calcified tissue was detected not only on the surface of the scaffold but also inside, this attesting to the 


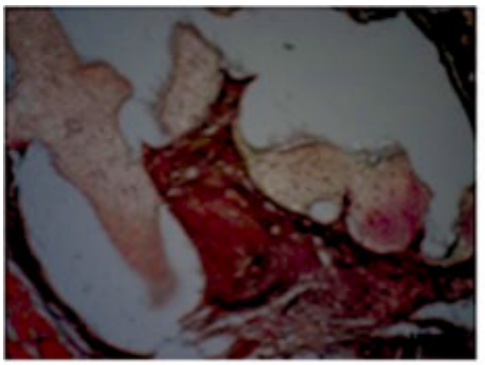

A

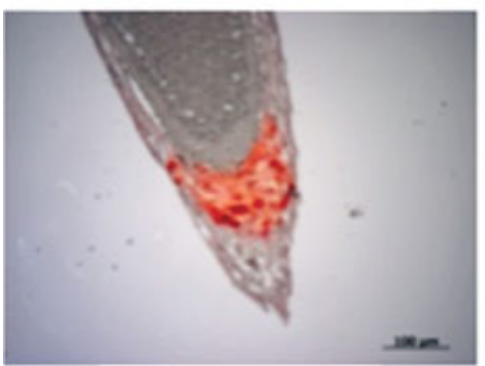

$\mathrm{C}$

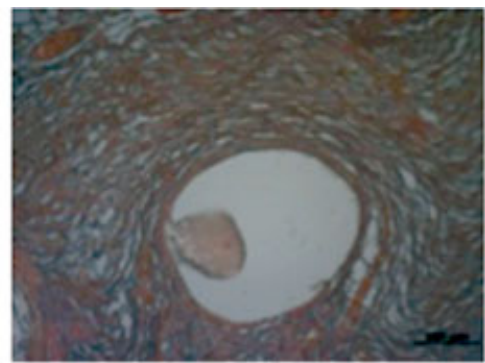

B

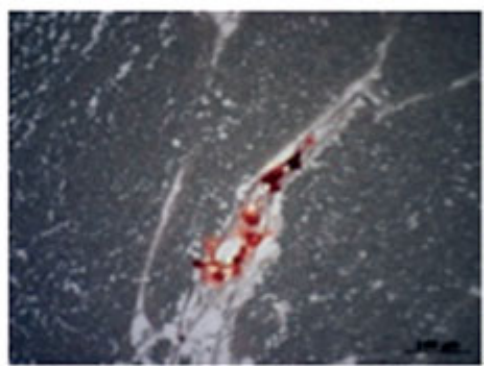

D

Figure 3. Optical microscopy pictures showing slide sections of the constructs seeded/cultured with the two hASCs populations considered and either implanted or in vitro cultured under different conditions and stained with alizarin red S. (A) ASCs ${ }^{\mathrm{CD} 29+}$ seeded in SPCL scaffolds implanted in nude mice $(\times 100)$; (B) ASCs ${ }^{\text {STO- }}{ }^{-1+}$ seeded in SPCL scaffolds implanted in nude mice $(\times 100)$; (C) ASCs ${ }^{\mathrm{CD} 29+}$ seeded in SPCL scaffolds cultured in vitro with osteogenic medium $(\times 100)$; (D) ASCs ${ }^{\text {STRO-1+ }}$ seeded in SPCL scaffolds cultured in vitro with osteogenic medium

homogeneous distribution of cells into the scaffolds and their active metabolism and osteogenic differentiation in the whole volume of the construct. Finally, the $\mu \mathrm{CT}$ analysis further confirms the results obtained from real time RT-PCR and from the histological analysis, i.e. the higher osteogenic potential of constructs containing hASCs ${ }^{\text {STRO }-1+}$.

\section{Discussion}

The present study allowed comparison of two selected hASC subpopulations previously studied (Rada et al., 2010) and to clarify the behaviour of these cells, particularly their in vivo and in vitro osteogenic differentiation potentials, when seeded into SPCL fibre-mesh scaffolds, a well-characterized 3D structure (Gomes et al., 2003, 2008; Fuchs et al., 2009a; da Silva et al., 2009; Jukola et al., 2008). In order to trace the eventual cell migration, the cells used for the experiments were transfected with GFP. The detection of fluorescent cells in sections obtained from different samples confirms that most of the ASCs initially seeded in the scaffold were found in the original site of implantation of the construct, which allows direct correlation of the results obtained for the constructs that were implanted in nude mice to human ASCs and not to mouse stem cells that eventually could have migrated into the implant site.

The histological stainings and immunohistochemistry analyses showed that a mineralization process was ongoing in all the groups of samples considered, although the samples retrieved from nude mice showed a higher coloration when stained with alizarin red and higher fluorescence when stained with xylenol orange. Also regarding the immunohistochemistry for collagen I and osteopontin, it was possible to observe enhanced staining in samples retrieved from in vitro cultures, as compared to those that were implanted in nude mice. In fact, in the ASCs cultured in SPCL scaffolds in vitro with basal medium no posively stained regions were found, while ASCs cultured in SPCL scaffolds in vitro with osteogenic medium show some positive staining of the two osteogenic markers considered.

The results obtained with histological assays were confirmed by real-time RT-PCR. In fact the hASCs ${ }^{\mathrm{CD} 29+}$ scaffold constructs retrieved from the in vivo implants show an higher osteopontin expression when compared with the in vitro-cultured constructs containing the same hASCs subpopulation, while all the expression levels of the other genes considered is higher in the hASCs ${ }^{\mathrm{CD} 29+}$ - scaffold constructs resulting from the in vitro cultures. Regarding the constructs with the hASCs ${ }^{\text {STRO-1+ }}$, it was possible to observe a completely different scenario: in fact, all the osteogenic gene markers considered were more highly expressed in the constructs resulting from the implantation in nude mice compared with those retrieved from in vitro cultures. It is also important to highlight that the results obtained showed that all the samples cultured in 3D, i.e. all cells that were seeded/cultured onto the SPCL fibre-mesh scaffolds, exhibited higher osteogenic gene expression levels than cells cultured in 2D (well-plates; data not shown), demonstrating the importance of an adequate $3 \mathrm{D}$ environment in the development of the 


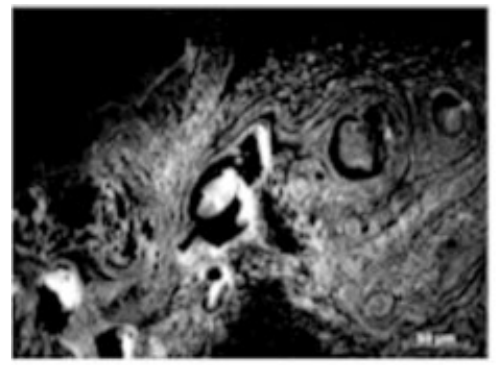

A

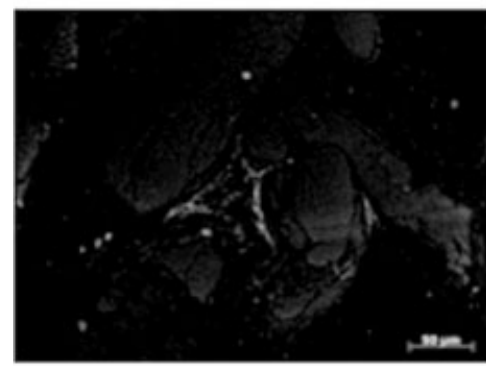

$\mathrm{C}$

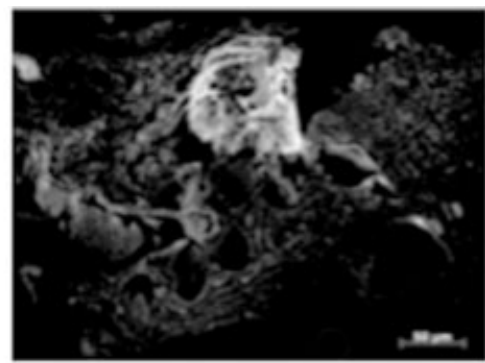

B

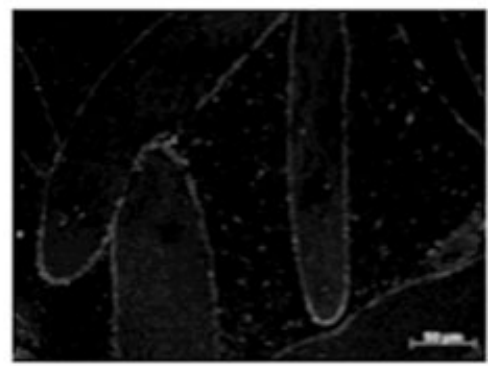

$\mathrm{D}$

Figure 4. Optical microscopy pictures showing slide sections of the constructs seeded/cultured with the two hASCs populations considered and either implanted or in vitro cultured under different conditions and stained for xylenol orange. (A) ASCs ${ }^{\mathrm{CD} 29+}$ seeded in SPCL scaffolds implanted in nude mice $(\times 100)$; (B) ASCs ${ }^{\text {STRO-1+ }}{ }^{-}$seeded in SPCL scaffolds implanted in nude mice $(\times 100)$; (C) ASCs ${ }^{\mathrm{CD} 29+}$ seeded in SPCL scaffolds cultured in vitro with osteogenic medium ( $\left.\times 100\right)$; (D) ASCs ${ }^{\text {STRO- } 1+}$ seeded in SPCL scaffolds cultured in vitro with osteogenic medium

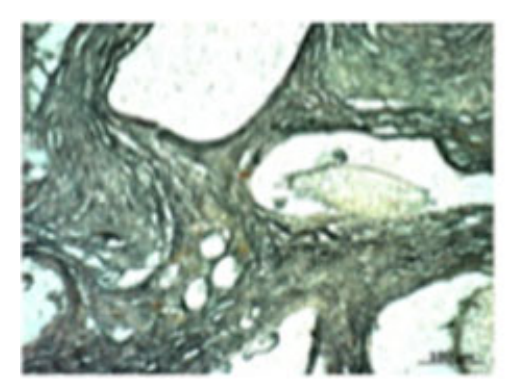

A

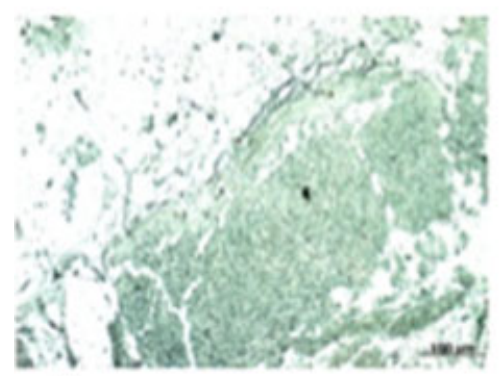

$\mathrm{C}$

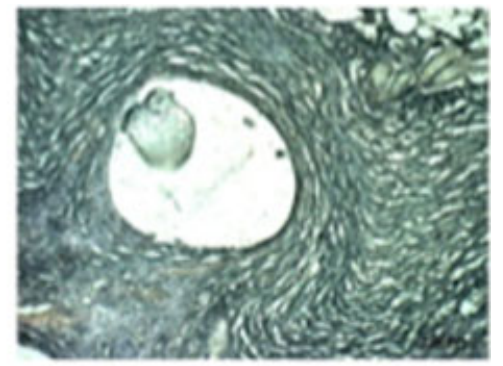

B

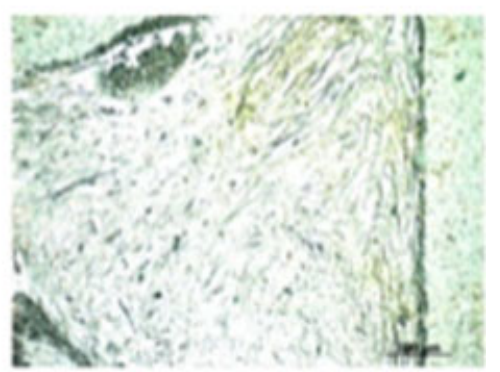

D

Figure 5. Optical microscopy pictures showing sections of the constructs seeded/cultured with the two hASCs populations considered and either implanted or in vitro cultured under different conditions and immunostained for osteopontin. (A) ASCs ${ }^{\mathrm{CD} 29+}$ seeded in SPCL scaffolds implanted in nude mice $(\times 100)$; (B) ASCs ${ }^{\text {TRO- }}{ }^{-1+}$ seeded in SPCL scaffolds implanted in nude mice $(\times 100)$; (C) ASCs ${ }^{\text {CD29+ }}$ seeded in SPCL scaffolds cultured in vitro with osteogenic medium $(\times 100)$; (D) ASCs ${ }^{\text {STRO- }-1+}$ seeded in SPCL scaffolds cultured in vitro with osteogenic medium

osteoblastic differentiation process and subsequent new bone formation.

Particularly interesting are the results obtained for constructs implanted in vivo that were previously seeded/cultured with the hASCs ${ }^{\text {TRO-1+ }}$ population, when compared to constructs seeded/cultured with the
hASCs ${ }^{\mathrm{CD} 29+}$; in fact, ASCs isolated with immunomagnetic beads coated with anti-STRO-1 antibody exhibited a much higher gene expression than those isolated with immunomagnetic beads coated with anti-CD29 antibody, demonstrating that the hASCs ${ }^{S T R O}-1+$ population is in a more advanced osteogenic differentiation stage than the 


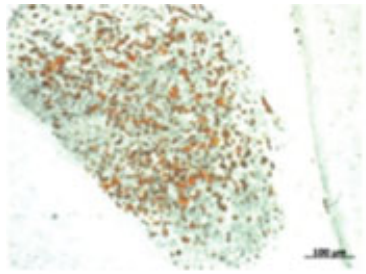

A

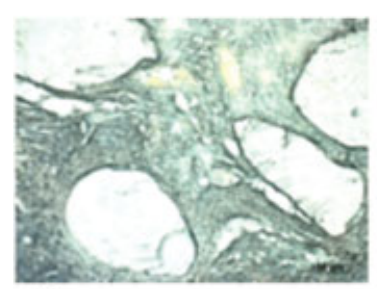

$\mathrm{D}$

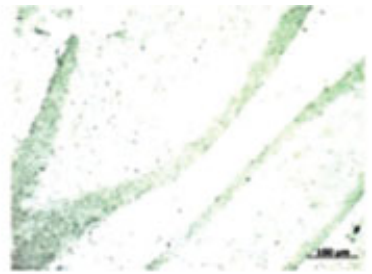

B

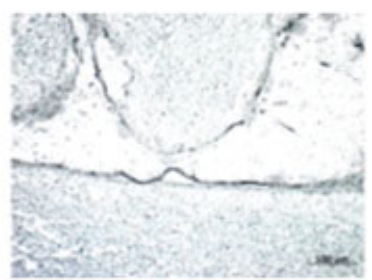

$\mathrm{E}$

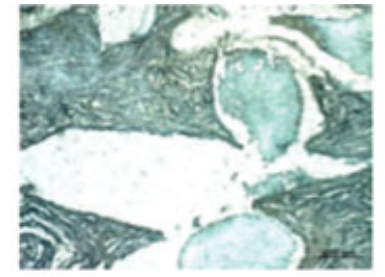

$\mathrm{C}$

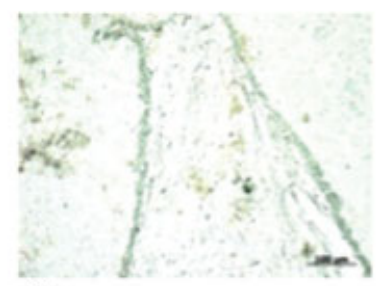

$\mathrm{F}$

Figure 6. Optical microscopy pictures showing sections of in the constructs seeded/cultured with the two hASCs populations considered and either implanted or in vitro cultured under different conditions and immunostained for collagen I. (A) ASCs ${ }^{\mathrm{CD} 29+}$ seeded in SPCL scaffolds cultured in vitro with basal medium $(\times 100)$; (B) ASCs ${ }^{\text {STRO }-1+}$ seeded in SPCL scaffolds cultured in vitro with basal medium $(\times 100)$; (C) ASCs ${ }^{\text {CD29+ }}$ seeded in SPCL scaffolds implanted in nude mice $(\times 100)$; (D) ASCs ${ }^{\text {STRO }-1+}$ seeded in SPCL scaffolds implanted in nude mice $(\times 100)$; (E) ASCs ${ }^{\mathrm{CD} 29+}$ seeded in SPCL scaffolds cultured in vitro with osteogenic medium ( $\times 100)$; (F) ASCs ${ }^{\text {STRO-1+ }}$ seeded in SPCL scaffolds cultured in vitro with osteogenic medium
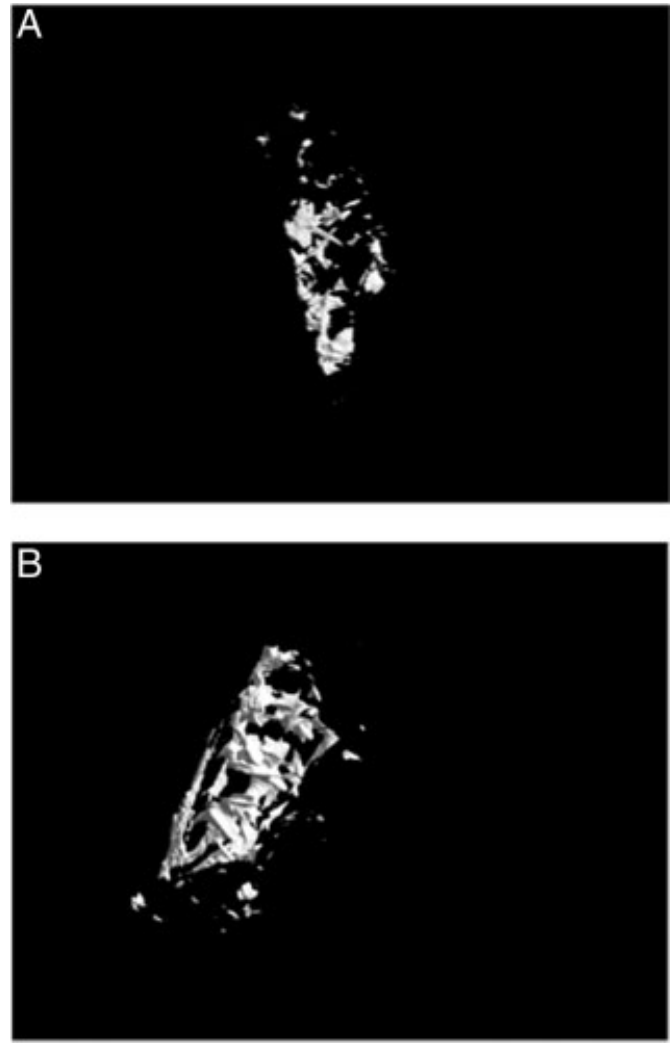

Figure 7. $\mu$ CT 3D virtual models images of SPCL scaffolds seeded with hASCs subpopulation and implanted in nude mice. (A) Scaffolds seeded with ASCs ${ }^{\mathrm{CD} 29+}$; (B) scaffolds seeded with ASCs $^{\text {STRO }}$

other ASCs population considered, data that were also confirmed by the amount of mineralization detected by $\mu \mathrm{CT}$ analysis.

Analysing the results obtained from the hASCs ${ }^{\text {STRO }-1+}$ population implanted in vivo, is possible to see a high expression of osterix, a gene that stimulates osteogenic differentiation and prevents chondrogenic differentiation (Caetano-Lopez et al., 2007) and that is essential to reach the status of osteoprogenitor cells (Ellies and Krumlauf, 2006; Stains and Civetelli, 2003). Contemporary to osterix, runx-2 is also highly expressed; in the hASCs ${ }^{\text {STRO }-1+}$ population implanted in vivo, the expression of this osteogenic differentiation gene is the highest of all the groups studied. Runx2 plays a critical role in osteogenic differentiation, particularly at the beginning of this process, contributing to the differentiation of mesenchymal stem cells to osteoprogenitor cells as well as in the differentiation of osteoprogenitor cells to osteoblasts (Ellies and Krumlauf, 2006; Stains and Civetelli, 2003; Karsenty, 2001). Considering that, in the case of hASCs ${ }^{\mathrm{STRO}-1+}$, besides the high levels of Runx-2, high expression of osteopontin and especially osteocalcin has also been detected, suggesting that the differentiation process is in the final stage and the cells implanted are almost differentiated into osteoblasts.

The hASCs ${ }^{\mathrm{CD} 29+}$ population implanted in vivo presents a different scenario regarding gene expression; in fact the massive overexpression of osterix compared with the other genes considered leads to the conclusion that these cells may have reached the status of osteoprecursor but are still not yet at that stage of differentiation that identifies them as osteoblasts.

Finally, when the results of cells implanted in vivo are compared with those cultured in vitro, it is possible to report two important findings. First, the expression levels of all genes studied obtained from in vivo and in vitro culture are either similar to or lower in the in vitro cultured constructs, underlining the role of an adequate physiological context (the in vivo environment) in which cells can sense several stimuli, not found in vitro, that promote the overexpression of the genes involved in 
osteogenic differentiation. Considering the position of the implants (subcutaneous) and the tissue surrounding the scaffold-cell constructs (mostly highly vascularized adipose tissue), most likely the factors stimulating the differentiation are VEGF (Maes et al., 2004; Zeng et al., 2007) and PPAR- $\gamma$ or leptin (Gimble et al., 2006). Second, the two ASC subpopulations considered, which were selected based on their osteogenic potential assessed in a previous in vitro study, showed different behaviour in vitro and in vivo, i.e. hASCs ${ }^{\text {TRO-1+ }}$ clearly showed higher osteogenic differentiation potential in vivo, while hASCs ${ }^{\mathrm{CD} 29+}$ demonstrated a good osteogenic differentiation potential in vitro, but not as good in vivo. The differences found in the osteogenic differentiation potential according to the cell subpopulation considered provides further evidence of the limitations of in vitro models, when the aim is to understand the behaviour of hASCs in a physiological environment.

Overall, the results obtained from this study allowed us to conclude that human adipose-derived stem cells isolated using anti STRO-1 ab have a great osteogenic potential and may enable a more efficient use of adipose tissue as a cell source for bone tissue-engineering applications. The data showed also confirm the importance of the physiological context and also of the 3D environment provided by an adequate scaffold, specifically the SPCL fibre-meshes, to enhance bone regeneration through TE therapies. Despite the importance of the identification of a subpopulation of ASCs with great potential for osteogenic differentiation, further studies will be crucial to determine the correlation between STRO-1 and osteogenic differentiation and, globally, the role of STRO-1-positive stem cells in the different niches of the human body.

\section{References}

Caetano-Lopes J, Canhao H, Fonseca JE. 2007; Osteoblasts and bone formation. Acta Reumatol Port 32(2): 103-110.

da Silva MA, Crawford A, Mundy J, et al. 2009; Evaluation of extracellular matrix formation in polycaprolactone and starchcompounded polycaprolactone nanofiber meshes when seeded with bovine articular chondrocytes. Tissue Eng A 15(2): 377-385.

Ellies DL, Krumlauf R. 2006; Bone formation: the nuclear matrix reloaded. Cell 125(5): 840-842.

Fraser JK, Schreiber R, Strem B, et al. 2006; Plasticity of human adipose stem cells toward endothelial cells and cardiomyocytes. Nat Clin Pract Cardiovasc Med 3(suppl 1): S33-37.

Fuchs S, Ghanaati S, Orth C, et al. 2009a; Contribution of outgrowth endothelial cells from human peripheral blood on in vivo vascularization of bone tissue engineered constructs based on starch polycaprolactone scaffolds. Biomaterials 30(4): 526-534.

Fuchs S, Jiang X, Schmidt H, et al. 2009b; Dynamic processes involved in the prevascularization of silk fibroin constructs for bone regeneration using outgrowth endothelial cells. Biomaterials $\mathbf{3 0}(7)$ : 1329-1338.

Fujimura J, Ogawa R, Mizuno H, Fukunaga Y, Suzuki H. 2005; Neural differentiation of adipose-derived stem cells isolated from GFP transgenic mice. Biochem Biophys Res Commun 333(1): 116-121.

Gay IC, Chen S, MacDougall M. 2007; Isolation and characterization of multipotent human periodontal ligament stem cells. Orthodont Craniofac Res 10(3): 149-160.

Gimble J, Guilak F. 2003a; Adipose-derived adult stem cells: isolation, characterization, and differentiation potential. Cytotherapy 5(5): 362-369.
Gimble JM, Guilak F. 2003b; Differentiation potential of adipose derived adult stem (ADAS) cells. Curr Top Dev Biol 58: 137-160.

Gimble JM, Zvonic S, Floyd ZE, Kassem M, Nuttall ME. 2006; Playing with bone and fat. J Cell Biochem 98(2): 251-266.

Gomes ME, Reis RL. 2004; Tissue engineering: key elements and some trends. Macromol Biosci 4(8): 737-742.

Gomes ME, Sikavitsas VI, Behravesh E, Reis RL, Mikos AG. 2003; Effect of flow perfusion on the osteogenic differentiation of bone marrow stromal cells cultured on starch-based three-dimensional scaffolds. $J$ Biomed Mater Res A 67(1): 87-95.

Gomes ME, Ribeiro AS, Malafaya PB, Reis RL, Cunha AM. 2001a; A new approach based on injection moulding to produce biodegradable starch-based polymeric scaffolds: morphology, mechanical and degradation behaviour. Biomaterials 22(9): 883-889.

Gomes ME, Reis RL, Cunha AM, Blitterswijk CA, de Bruijn JD. 2001b; Cytocompatibility and response of osteoblastic-like cells to starch-based polymers: effect of several additives and processing conditions. Biomaterials 22(13): 1911-1917.

Gomes ME, Bossano CM, Johnston CM, Reis RL, Mikos AG. 2006a; In vitro localization of bone growth factors in constructs of biodegradable scaffolds seeded with marrow stromal cells and cultured in a flow perfusion bioreactor. Tissue Eng 12(1): 177-188.

Gomes ME, Holtorf HL, Reis RL, Mikos AG, et al. 2006b; Influence of the porosity of starch-based fiber mesh scaffolds on the proliferation and osteogenic differentiation of bone marrow stromal cells cultured in a flow perfusion bioreactor. Tissue Eng 12(4): 801-809.

Gomes ME, Azevedo HS, Moreira AR, Ellä V, Kellomäki M, Reis RL. 2008;
Starch-poly( $\varepsilon$-caprolactone) and starchpoly(lactic acid) fibre-mesh scaffolds for bone tissue engineering applications: structure, mechanical properties and degradation behaviour. $J$ Tissue Eng Regen Med 2(5): 243-252.

Hennrick KT, Keeton AG, Nanua S, et al. 2007; Lung cells from neonates show a mesenchymal stem cell phenotype. Am J Respir Crit Care Med 175(11): 1158-1164.

Huang JI, Kazmi N, Durbhakula MM, Hering TM, Yoo JU, Johnstone B. 2005; Chondrogenic potential of progenitor cells derived from human bone marrow and adipose tissue: a patient-matched comparison. J Orthop Res 23(6): 1383-1389.

Hui JH, Li L, Teo YH, Ouyang HW, Lee EH. 2005; Comparative study of the ability of mesenchymal stem cells derived from bone marrow, periosteum, and adipose tissue in treatment of partial growth arrest in rabbit. Tissue Eng 11(5-6): 904-912.

Jukola H, Nikkola L, Gomes ME, et al. 2008; Development of a bioactive glass fiber reinforced starch-polycaprolactone composite. J Biomed Mater Res B Appl Biomater 87(1): 197-203.

Karsenty G. 2001; Minireview: transcriptional control of osteoblast differentiation. Endocrinology 142(7): 2731-2733.

Lee JH, Kemp DM. 2006; Human adiposederived stem cells display myogenic potential and perturbed function in hypoxic conditions. Biochem Biophys Res Commun 341(3): 882-888.

Maes C, Stockmans I, Moermans K, et al. 2004; Soluble VEGF isoforms are essential for establishing epiphyseal vascularization and regulating chondrocyte development and survival. J Clin Invest 113(2): 188-199.

Majore I, Moretti P, Hass R, Kasper C. 2009; Identification of subpopulations in mesenchymal stem cell-like cultures from 
human umbilical cord. Cell Commun Signal 7: 6 .

Martins AM, Pham QP, Malafaya PB, et al. 2008; The role of lipase and $\alpha$-amylase in the degradation of $\operatorname{starch} / \operatorname{poly}(\varepsilon$ caprolactone) fiber meshes and the osteogenic differentiation of cultured marrow stromal cells. Tissue Eng A 15(2): 295-305.

Martins AM, Pham QP, Malafaya PB, et al. 2009; The role of lipase and $\alpha$-amylase in the degradation of $\operatorname{starch} / \operatorname{poly}(\varepsilon$ caprolactone) fiber meshes and the osteogenic differentiation of cultured marrow stromal cells. Tissue Eng A 15(2): 295-305.

McIntosh K, Zvonic S, Garrett S, et al. 2006; The immunogenicity of human adiposederived cells: temporal changes in vitro. Stem Cells 24(5): 1246-1253.

Mochizuki T, Muneta T, Sakaguchi Y, et al. 2006; Higher chondrogenic potential of fibrous synovium- and adipose synoviumderived cells compared with subcutaneous fat-derived cells: distinguishing properties of mesenchymal stem cells in humans. Arthritis Rheum 54(3): 843-853.

Ogawa R, Mizuno H, Watanabe A, Migita M Shimada T, Hyakusoku H. 2004; Osteogenic and chondrogenic differentiation by adipose-derived stem cells harvested from GFP transgenic mice. Biochem Biophys Res Commun 313(4): 871-877.

Oliveira JT, Crawford A, Mundy JM et al. 2007; A cartilage tissue engineering approach combining starchpolycaprolactone fibre mesh scaffolds with bovine articular chondrocytes. J Mater Sci Mater Med 18(2): 295-302.

Planat-Benard V, Menard C, André M, et al. 2004; Spontaneous cardiomyocyte differentiation from adipose tissue stroma cells. Circ Res 94(2): 223-229.

Puissant B, Barreau C, Bourin P, et al. 2005; Immunomodulatory effect of human adipose tissue-derived adult stem cells: comparison with bone marrow mesenchymal stem cells. $\mathrm{Br} J$ Haematol 129(1): 118-129.
Rada T, Gomes ME, Reis RL. 2010; Distinct stem cells subpopulations isolated from human adipose tissue exhibit different chondrogenic and osteogenic differentiation potential. Stem Cell Rev. [Epub ahead of print]. PMID: 20396979.

Rada T, Reis RL, Gomes ME. 2009a; Adipose tissue-derived stem cells and their application in bone and cartilage tissue engineering. Tissue Eng B Rev 15(2): 113-125.

Rada T, Reis RL, Gomes ME. 2009b; Novel method for the isolation of adipose stem cells (ASCs). J Tissue Eng Regen Med 3(2): 158-159.

Safford KM, Hicok KC, Safford SD, et al. 2002; Neurogenic differentiation of murine and human adipose-derived stromal cells. Biochem Biophys Res Commun 294(2): 371-379.

Santos MI, Fuchs S, Gomes ME, Unger RE, Reis RL, Kirkpatrick CJ. 2007; Response of micro- and macrovascular endothelial cells to starch-based fiber meshes for bone tissue engineering. Biomaterials 28(2): 240-248.

Santos MI, Tuzlakoglu K, Fuchs S, et al. 2008; Endothelial cell colonization and angiogenic potential of combined nanoand micro-fibrous scaffolds for bone tissue engineering. Biomaterials 29(32): 4306-4313.

Silva GA, Coutinho OP, Ducheyne P, Reis RL. 2007; Materials in particulate form for tissue engineering. 2. Applications in bone. $J$ Tissue Eng Regen Med 1(2): 97-109.

Silva NA, Salgado AJ, Sousa RA, et al. 2009; Development and characterization of a novel hybrid tissue engineering based scaffold for spinal cord injury repair. Tissue Eng A 16(1): 45-54.

Stains J, Civitelli R. 2003; Genomic approaches to identifying transcriptional regulators of osteoblast differentiation. Genome Biol 4(7): 222

Stock P, Staege MS, Müller LP, et al. 2008; Hepatocytes derived from adult stem cells. Transpl Proc 40(2): 620-623.

Strem BM, Zhu M, Alfonso Z, et al. 2005; Expression of cardiomyocytic markers on adipose tissue-derived cells in a murine model of acute myocardial injury. Cytotherapy 7(3): 282-291.

Tuzlakoglu K, Bolgen N, Salgado AJ, Gomes ME, Piskin E, Reis RL. 2005; Nanoand micro-fiber combined scaffolds: a new architecture for bone tissue engineering. J Mater Sci Mater Med 16(12): 1099-1104.

Tuzlakoglu K, Pashkuleva I, Rodrigues MT, et al. 2009; A new route to produce starchbased fiber mesh scaffolds by wet spinning and subsequent surface modification as a way to improve cell attachment and proliferation. J Biomed Mater Res A 92(1): 369-377.

Xu Y, Malladi P, Wagner DR, Longaker MT. 2005; Adipose-derived mesenchymal cells as a potential cell source for skeletal regeneration. Curr Opin Mol Ther 7(4): 300-305.

Yamamoto Y, Banas A, Murata S, et al. 2008; A comparative analysis of the transcriptome and signal pathways in hepatic differentiation of human adipose mesenchymal stem cells. FEBS $J$ 275(6): 1260-1273.

Yoshimura H, Muneta T, Nimura A, Yokoyama A, Koga H, Sekiya I. 2007; Comparison of rat mesenchymal stem cells derived from bone marrow, synovium, periosteum, adipose tissue, and muscle. Cell Tissue Res 327(3): 449-462.

Zeng Q, Li X, Beck G, Balian G, Shen FH 2007; Growth and differentiation factor-5 (GDF-5) stimulates osteogenic differentiation and increases vascular endothelial growth factor (VEGF) levels in fatderived stromal cells in vitro. Bone 40(2): 374-381.

Zuk PA, Zhu M, Mizuno H, et al. 2001; Multilineage cells from human adipose tissue: implications for cell-based therapies. Tissue Eng 7(2): 211-228.

Zuk PA， Zhu M, Ashjian P, et al. 2002 Human adipose tissue is a source of multipotent stem cells. Mol Biol Cell 13(12): 4279-4295. 